\title{
A study of magnetic drug delivery system using bulk high temperature superconducting magnet
}

\section{$\operatorname{AUTHOR}(S)$ :}

Nishijima, Shigehiro; Takeda, Shin-ichi; Mishima, Fumihito; Tabata, Yasuhiko; Yamamoto, Masaya; Joh, Jun-ichiro; Iseki, Hiroshi; ... Sasaki, Akira; Jun, Kubota; Saho, Norihide

\section{CITATION:}

Nishijima, Shigehiro ...[et al]. A study of magnetic drug delivery system using bulk high temperature superconducting magnet. IEEE TRANSACTIONS ON APPLIED SUPERCONDUCTIVITY 2008, 18(2): 874-877

\section{ISSUE DATE:}

2008-06

URL:

http://hdl.handle.net/2433/84562

\section{RIGHT:}

(c) 2008 IEEE. Personal use of this material is permitted. However, permission to reprint/republish this material for advertising or promotional purposes or for creating new collective works for resale or redistribution to servers or lists, or to reuse any copyrighted component of this work in other works must be obtained from the IEEE. 


\section{A Study of Magnetic Drug Delivery System Using Bulk High Temperature Superconducting Magnet}

Shigehiro Nishijima, Shin-ichi Takeda, Fumihito Mishima, Yasuhiko Tabata, Masaya Yamamoto, Jun-ichiro Joh, Hiroshi Iseki, Yoshihiro Muragaki, Akira Sasaki, Kubota Jun, and Norihide Saho

\begin{abstract}
A magnetic drug delivery system (MDDS) has been studied to navigate and/or accumulate the magnetic seeded drug at a local diseased part in the human body. The bulk high temperature superconductors (HTS) are employed to control the drug because they would produce the required magnetic fields for MDDS. The trajectory of the ferromagnetic particles in the blood vessel was calculated and the possibility of the navigation of the drug is discussed. In the experiment the HTS $(\mathrm{GdBaCuO})$ which produces the magnetic field of $4.5 \mathrm{~T}$ at the surface, was located $25 \mathrm{~mm}$ apart from the Y-shaped blood vessel. The drug navigation probability to the desired direction was confirmed to be higher than $80 \%$. A rat experiment was also performed successfully using a permanent magnet. The possible MDDS system will be discussed.
\end{abstract}

Index Terms-Bulk high temperature superconductor, magnetic drug delivery system, magnetic seeded drug, nano-sized particle, navigation of drag.

\section{INTRODUCTION}

W HEN the drug delivery system that accurately transports the drug of the proper quantity to the diseased part is used, the reduction of the dosage and the side effect can be expected more than the conventional administering technique. Moreover, it is thought that the treatment of a difficult part of a surgical approach becomes possible, and an effective treatment method to an obstinacy disease is achieved. We decided to apply the technique of the particle motion control by magnetic force to the DDS [1]-[3].

Magnetic force to the magnetic seeded drug with the ferromagnetic fine particle is induced by a superconducting bulk magnet. The magnet is placed outside of the body. The magnetic seeded drug is injected into a blood vessel and is navigated to the diseased part by the magnetic force. The applicability of the MDDS has been studied [4], [5].

Manuscript received August 25, 2007. This work was supported in part by NEDO under R/D Project on Next-Generation DDS Treatment Systems for Malignant Tumors, R/D on Next-Generation DDS Treatment Systems for Deep Therapy.

S. Nishijima, S. Takeda, and F. Mishima are with the Division of Sustainable Energy and Environmental Engineering, Osaka University, Osaka 565-0871, Japan (e-mail: nishijim@see.eng.osaka-u.ac.jp; stakeda@see.eng.osaka-u.ac.jp).

Y. Tabata, M. Yamamoto, and J. Joh are with the Department of Biomaterials, Field of Tissue Engineering, Institute for Frontier Medical Science, Kyoto University, Japan.

H. Iseki and Y. Muragaki are with the Faculty of Advanced Techno-Surgery, Institute of Advanced Biomedical Engineering and Science, Graduate School of Medicine, Department of Neurosurgery, Neurological Institute, Tokyo Women's Medical University, Japan.

A. Sasaki and J. Kubota are with Hitachi Medical Corporation, Japan.

N. Saho is with Hitachi, Ltd., Japan.

Digital Object Identifier 10.1109/TASC.2008.921967

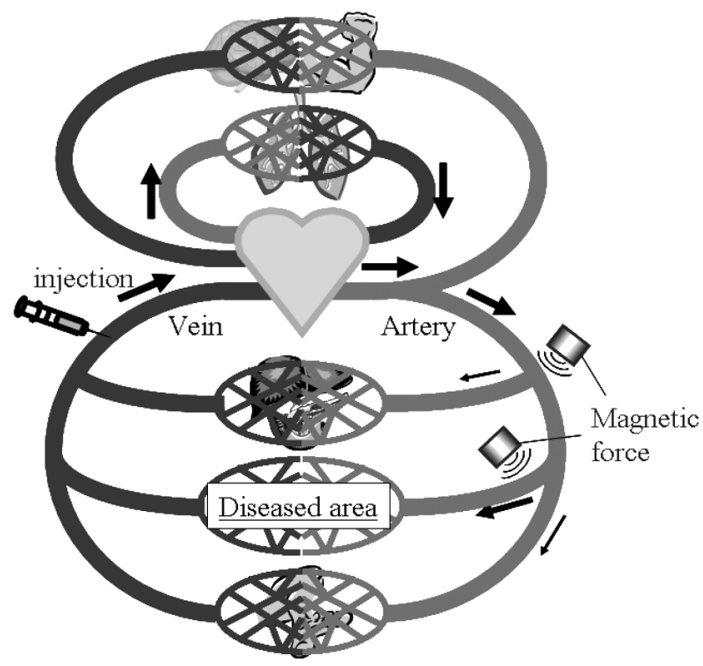

Fig. 1. Course that sends a drug to a diseased part in MDDS.

Because the drug can be controlled by the magnetic force in the body, the achievement of a high concentration of the drug in a target diseased part. The reduction of toxicity to the normal tissue with the drug could be achieved and hence MDDS is one of promising DDS. In this work the possibility of MDDS was examined. The animal test [6] was also performed using rat.

\section{CONCEPT OF MDDS}

The following methods were devised as a method of MDDS. The drug is conveyed by the current of blood and is delivered. The magnet is put on the surface of the body near the branching point of the blood vessel. The drug is delivered in the direction of the purpose by the magnetic force. The drug is finally delivered to a targeted diseased part by repeating this navigation.

When this concept is actually used, the following procedure will be made. First, the magnetic seeded drug is introduced into the inside of the body by the vein injection. The drug goes from the vein to the heart (right atrium), passed pulmonary artery from the right ventricle, and circulates to lungs, the pulmonary vein, and the heart (left atrium). Then blood spreads in the whole body from the left ventricle through the aorta as shown in Fig. 1. The magnetic seeded drug that has come out from the left ventricle is navigated in a specific direction with the superconducting magnet placed outside of the body near the branching point of the blood vessel. This navigation is repeated two or more times. Moreover, the magnet is placed in the diseased part and hence the drug is accumulated at the diseased part by the magnetic force [7]-[9]. To pass the capillary of lungs and to 


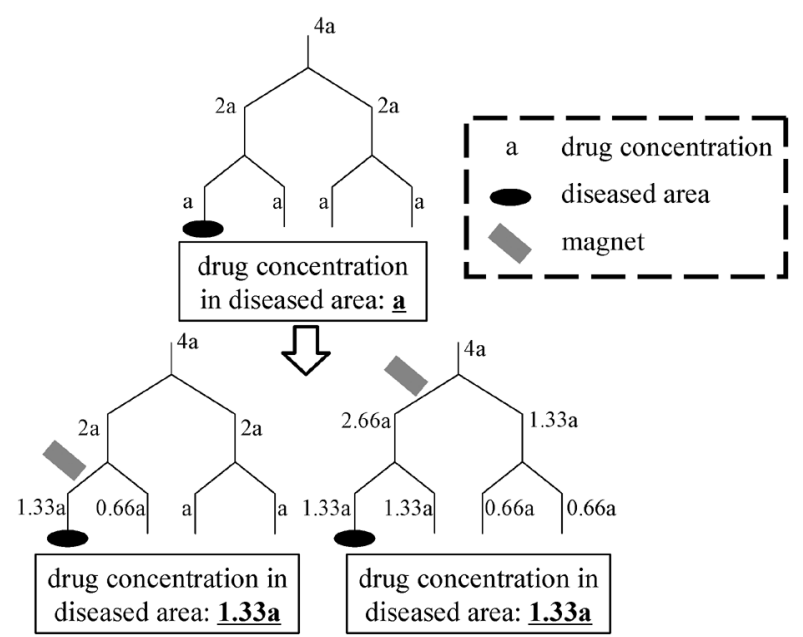

Fig. 2. Effect of the branching point on the drug concentration in the diseased part with navigation rate of $2: 1$.

prevent the phagocytosis, a drug of $100 \mathrm{~nm}$ or less is said to be preferable.

The necessary navigation efficiency was calculated. The concentration of the drug in blood after navigation is shown schematically in Fig. 2. It is assumed that the diseased part exists after branching two times of blood vessel. When the drug concentration in blood before branching is assumed to be $4 \mathrm{a}$, the concentration after two times branching becomes a. Hence, the concentration of the drug in the diseased part is a.

When the navigation rate is set to $2: 1$ at the 2 nd branching point, the ratio of drug concentration in blood is 1.33a:0.66a after the 2nd branching. The improvement of the concentration of the drug in the diseased part is about $33 \%$.

When the navigation rate is set to $2: 1$ at the 1 st branching point, the ratio of drug concentration in blood is 2.66a:1.33a after the first branching. After 2nd branching the concentration of the drug becomes 1.33a or 0.66a. It means that the blood having $33 \%$ increase of drug concentration is admitted in the diseased part.

When the navigation ratio is adjusted to $2: 1$ upstream of the diseased part, the concentration of the drug is increased by $33 \%$ in the diseased part. In practical use of MDDS the $30 \%$ improvement of the drug concentration can be expected in the diseased part if the navigation ratio is set 2:1 upstream of the diseased part, where the navigation is easily made (that is the blood vessel near the body surface or vessel density is a little). When the navigation efficiency is adjusted to $4: 1$, a concentration increase of about $60 \%$ will be admitted. The tentative target of this research was assumed to adjust the navigation ratio $2: 1$ or more.

\section{NAVigation With BUlk Superconducting MagneT}

The following 2 subjects were examined in the study.

1) Using magnetite particle of $100 \mathrm{~nm}$ in the diameter, possibility of the navigation rate of 2:1 is examined with water of $200 \mathrm{~mm} / \mathrm{s}$ in a glass tube placed away from the magnet by $50 \mathrm{~mm}$. 2) Using $100 \mathrm{~nm}$ magnetite particle, the possibility of the navigation rate of $2: 1$ is examined with blood of $200 \mathrm{~mm} / \mathrm{s}$ in a blood vessel placed away from the magnet by $25 \mathrm{~mm}$.

Since there were a lot of discussions about the magnetic navigation of nano-sized particle, to clarify the condition that is able

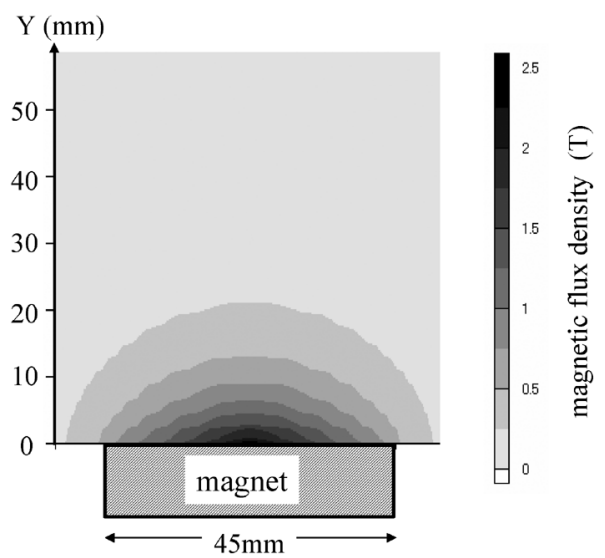

Fig. 3. Magnetic field generated by a bulk superconducting magnet.

to be navigated quantitatively, the calculation and the experiment were made.

\section{A. Particle Trajectory in Magnetic Field}

A calculation was made to see if the required magnetic fields can be generated by the bulk superconducting at deep inside of body. The flow velocity distribution in the glass tube and the magnetic field distribution around the magnet were analyzed by using finite element method (FEM). Then the particle trajectory in the magnetic field was calculated by using the results of FEM.

The particle trajectories are calculated according to the following procedures.

Water was thrown into the straight glass tube of $2 \mathrm{~mm}$ in the inner diameter at the speed of $200 \mathrm{~mm} / \mathrm{s}$. Several magnetite particles of which the diameter is $100 \mathrm{~nm}$ were put in this. As an initial condition, the speed of the particle was set as the drag force to the particle becomes 0 . Magnetic force $F_{M}$ and drag force $F_{D}$ from water to the particle are calculated as (1) and (2).

$$
\begin{aligned}
F_{M} & =\frac{4}{3} \cdot b^{3} \cdot \mu_{0} \frac{9\left(\chi_{p}-\chi_{f}\right)}{\left(3+\chi_{p}\right) \cdot\left(3+\chi_{f}\right)} H g r a d H \\
F_{D} & =6 \pi \cdot \eta \cdot b \cdot\left(v_{f}-v_{p}\right)
\end{aligned}
$$

Here, $\chi_{p}$ and $\chi_{f}$ are volume magnetic susceptibility of the particle and the fluid. The $v_{p}$, and $v_{f}$ are the speeds of the particle and the fluid, respectively. The $\eta$ is the viscosity of the fluid, $b$ the particle diameter, $H$ strength of the magnetic field. As the difference between $F_{M}$ and $F_{D}$ is the force to the particle, the equation of motion of the particle was solved with time evolution. The viscosity of water were assumed to be $1.0 \times 10^{-3} \mathrm{~Pa} \cdot \mathrm{s}$. In the calculation up to $0.5 \mathrm{~T}$ the intensity of magnetization was proportional to magnetic field (the volume magnetic susceptibility was 8000), and then came to be constant as saturated magnetization $(0.6 \mathrm{~T})$.

Fig. 3 shows the contour line of the magnetic field generated by the bulk superconducting magnet. A disk shaped balk superconductor of which dimension was $40 \mathrm{~mm}$ in diameter and $15 \mathrm{~mm}$ in thickness was used in the calculation assuming the critical current density as $5 \times 10^{8} \mathrm{~A} / \mathrm{m}^{2}$. The magnetic flux density at the surface of the magnet was $4.5 \mathrm{~T}$. The critical current density was set to reproduce the measured magnetic flux 


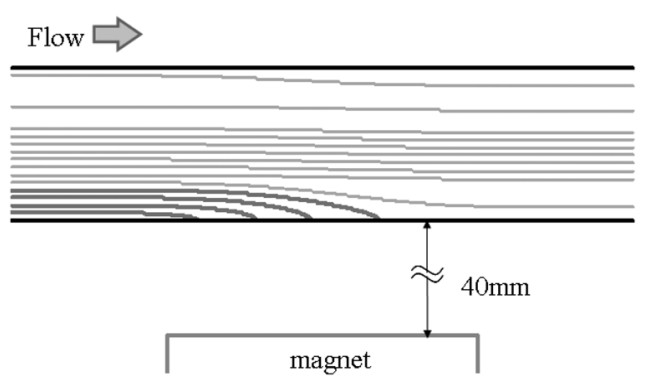

Fig. 4. Trajectories of the particle in a glass tube put to the distance of $40 \mathrm{~mm}$.

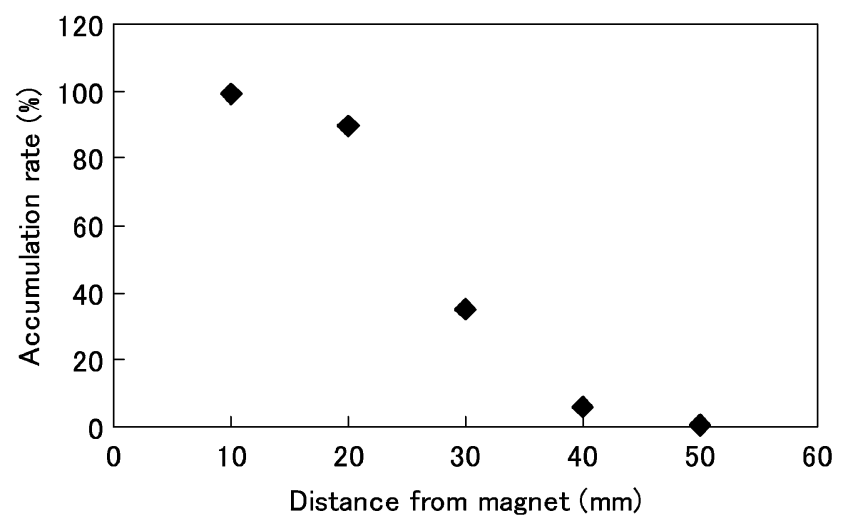

Fig. 5. A change of the accumulation rate with the distance.

density. The particle trajectories of the ferromagnetic particles in water were calculated in the magnetic filed generated by the bulk superconducting magnet.

Fig. 4 shows calculated results of particle trajectories. The glass tube was located at $40 \mathrm{~mm}$ above the bulk superconducting magnet. It was understood that even at the distance of $40 \mathrm{~mm}$ above the magnet the particles can be accumulated. The accumulated fraction was calculated by changing the distance between glass tube and the magnet assuming the parabolic flow velocity distribution and shown in Fig. 5. This suggests that the particle can be accumulated up to $50 \mathrm{~mm}$ distance. In the blood the higher magnetic field would be necessary for the accumulation.

\section{B. Navigation From a Long Distance}

Based on the calculation, the navigation experiments were made in water $50 \mathrm{~mm}$ away and in blood $25 \mathrm{~mm}$ away from the magnet. The blood and blood vessel taken from pigs were used. The branching point of glass tube or blood vessel was placed above $50 \mathrm{~mm}$ or $25 \mathrm{~mm}$ from the surface of the bulk magnet.

The distilled water was thrown at the flow velocity of $200 \mathrm{~mm} / \mathrm{s}$ in the Y-shaped glass tube of which inside diameter is $2 \mathrm{~mm}$. The branching point of glass tube was placed above $50 \mathrm{~mm}$ from the surface of the bulk magnet. The suspension was injected into the tube before the branching point and the navigation efficiency after branching was measured.

For preparing the suspension the magnetite particle of $100 \mathrm{~nm}$ in diameter was dispersed in water. The particle size distribution was measured to see if the particle aggregate or not. The dispersed particle was confirmed not to aggregate in water.

The magnetic flux density of the bulk superconducting magnet used for the navigation is $4.5 \mathrm{~T}$ on the surface of

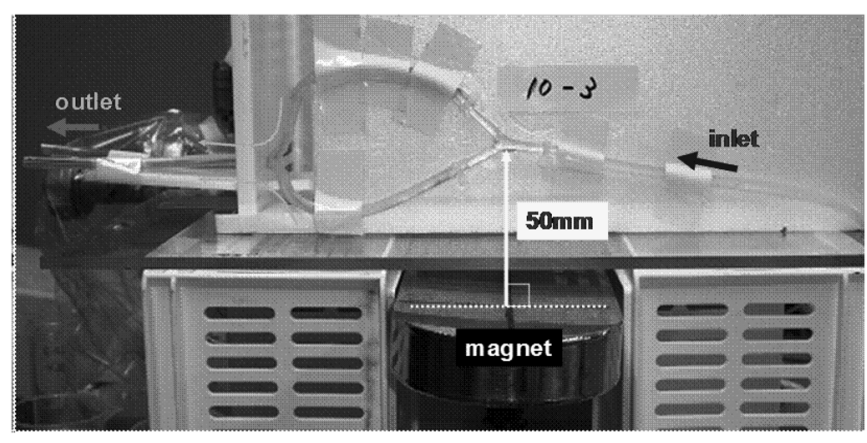

Fig. 6. Appearance of the experimental set up.

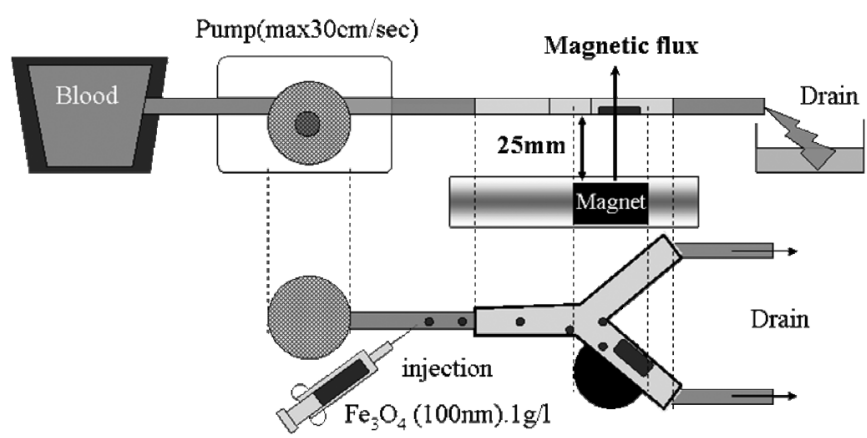

Fig. 7. Schematic view of the navigation at branching point by a bulk superconducting magnet.

the magnet. A magnetic field and a magnetic gradient at the Y-shaped glass tube were $0.088 \mathrm{~T}$ and $4.3 \mathrm{~T} / \mathrm{m}$, respectively. The navigation efficiency was measured with a magnetic balance. The photograph of the experimental set up is shown in Fig. 6 . The ferromagnetic particle was navigated to downward in the experiment. It was examined whether the navigation rate can be more than 2:1.

It was successfully confirmed that the navigation rate was 66.9:33.1.

The navigation experiment was made by using the blood of pig and the branching point of blood vessel in abdominal aorta of pig. The bulk superconducting magnet was also used as a magnetic field source. The schematic illustration of the experimental set up was shown in Fig. 7. The blood vessel was kept horizontally and parallel to the surface of the magnet, and was placed at $25 \mathrm{~mm}$ distance from the magnet. The blood was thrown in the blood vessel at the speed of $200 \mathrm{~mm} / \mathrm{s}$. The magnetite suspension was injected to the blood stream at the speed of $3 \mathrm{ml} / \mathrm{min}$. The magnetic field and the gradient of the magnetic field at the branching point were $0.3 \mathrm{~T}$ and $25 \mathrm{~T} / \mathrm{m}$, respectively.

Two blood vessels that were used are $4 \mathrm{~mm}$ and $3 \mathrm{~mm}$ in inner diameter. The obtained navigation rate was 86.9:13.1 (6.6:1) in $4 \mathrm{~mm}$ and 89.9:10.1 (8.9:1) in $3 \mathrm{~mm}$ blood vessel. In Fig. 8 the photos of Y-shaped blood vessel were shown.

It was confirmed by means of calculation and experiment that with the bulk superconducting magnet the navigation rate can attain 2:1 even at deep inside of the body.

\section{ANIMAL EXPERIMENT}

The animal experiment using rat was performed. As a Y-shaped blood vessel the abdominal aorta branching to right and left ileum artery was chosen. A permanent magnet of which 

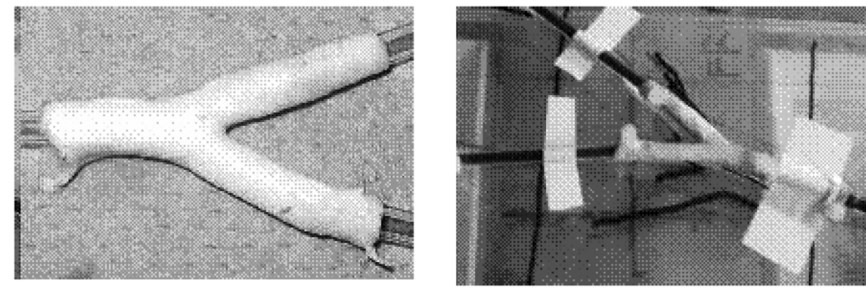

Fig. 8. Y-shaped blood vessel and navigation at branching point.

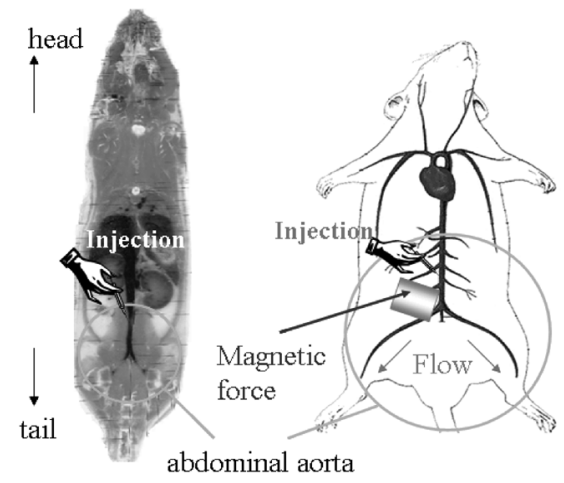

Fig. 9. Navigation experiment at branching point of blood vessel using rat and permanent magnet.

magnetic field was $0.3 \mathrm{~T}$ was put near the right ileum after branching. Magnetic capsules were injected into the abdominal aorta and the quantity of the capsules guided by a magnetic field was measured with a magnetic balance. In Fig. 9 the experimental method was shown. The concentration of the capsules was compared between the blood vessel with the magnet and the one without the magnet. The magnet increased the concentration of the capsule more than double. The navigation ratio was found to be 86.0:14.0.

\section{Head Cervical CANCER}

An advantageous part for a clinical application of MDDS was surveyed based on the obtained results. It was proposed that MDDS could be applied to skin cancer, bone tumors and head cervical cancer (nose, mouth, jaw, throat and ear), lung cancer, and liver cancer. Especially, high navigation efficiency can be expected to the skin cancer. Concerning lung cancer, as there are no surrounding internal organs, the local drug concentration can be increases by MDDS and hence MDDS could be a promising treatment method.

It was also pointed out that there was a possibility of becoming a treatment method that took the place of the artery injection chemotherapy about the head cervical cancer.

\section{CONCLUSION}

The concept of MDDS and the method to realize were examined. In the method the magnetic seeded drug was introduced into the inside of the body by the vein injection and navigated at the branching point of the artery to the diseased part. When the navigation efficiency was increased to $2: 1$ in the branching point, the concentration of the drug was shown to be improved by $30 \%$ in the downstream. The calculation method for the navigation of the magnetic seeded drug in the blood vessel by the magnetic field was developed. The calculation showed that the bulk superconducting magnet was possible to obtain the navigation efficiency of 2:1 at deep inside of the body of $50 \mathrm{~mm}$. The calculation result was actually confirmed by the experiment. The animal experiment was also performed successfully. The clinical applications with high possibility were surveyed based on the obtained results.

\section{ACKNOWLEDGMENT}

The authors would like to thank Prof. H. Fujishiro (Faculty of Engineering, Iwate University) for allowing us to use the bulk superconducting magnet.

\section{REFERENCES}

[1] F. Mishima, S. Takeda, Y. Izumi, and S. Nishijima, "Three dimensional motion control system of ferromagnetic particles for magnetically targeted drug delivery systems," IEEE Trans. Appl. Supercond., vol. 16, no. 2, pp. 1539-1542, 2006.

[2] F. Mishima, F. Fujimoto, S. Takeda, Y. Izumi, and S. Nishijima, "Development of control system for magnetically targeted drug delivery," J. Magnetism Magn. Mat., vol. 310, pp. 2883-2885, 2007.

[3] F. Mishima, S. Takeda, Y. Izumi, and S. Nishijima, "Development of magnetic field control for magnetically targeted drug delivery system using a superconducting magnet," IEEE Trans. Appl. Supercond., vol. 17, pp. 2303-2306, 2007.

[4] C. Alexious, W. Arnold, R. J. Klein, F. G. Parak, P. Hulin, C. Bergemann, W. Erhardt, S. Wagenpfeil, and A. S. Lubbe, "Locoregional cancer treatment with magnetic drug targeting," CANCER RESERCH, vol. 60, pp. 6641-6648, Dec. 1, 2000.

[5] C. Alexious, R. J. Schmid, R. Jurgons, M. Kremer, G. Wanner, C. Bergemann, E. Huenges, T. Nawroth, W. Arnold, and F. G. Parak, "Targeting cancer cells: Magnetic nanoparticles as drug carriers," Eur Biophys J, vol. 35, pp. 446-450, 2006.

[6] Y. Yoshida, S. Fukui, S. Fujimoto, F. Mishima, S. Takeda, Y. Izumi, S. Ohtani, Y. Fujitani, and S. Nishijima, "Ex vivo investigation of magnetically targeted drug delivery system," J. Magnetism Magn. Mat., vol. 310, pp. 2880-2882, 2007.

[7] S. Takeda, F. Mishima, B. Terazono, Y. Izumi, and S. Nishijima, "Development of magnetic force-assisted new gene transfer system using biopolymer-coated ferromagnetic nanoparticles," IEEE Trans. Appl. Supercond., vol. 16, no. 2, pp. 1543-1546, 2006.

[8] N. Morishita, H. Nakagami, R. Morishita, S. Takeda, F. Mishima, B. Terazono, S. Nishijima, Y. Kaneda, and N. Tanaka, "Magnetic nanoparticle with surface modification enhanced gene delivery of HVJ-E vector," Biochem. Biophys. Res. Commun., vol. 334, pp. 1121-1126, 2005.

[9] S. Takeda, B. Terazono, F. Mishima, H. Nakagami, S. Nishijima, and Y. Kaneda, "Novel drug delivery system by surface modified magnetic nanoparticles," J. Nanoscience and Nanotechnology, vol. 6, pp. 3269-3276, 2006. 